\title{
Política social, pobreza y focalización
}

DOI: 10.22403/UQROOMX/TYP04/01

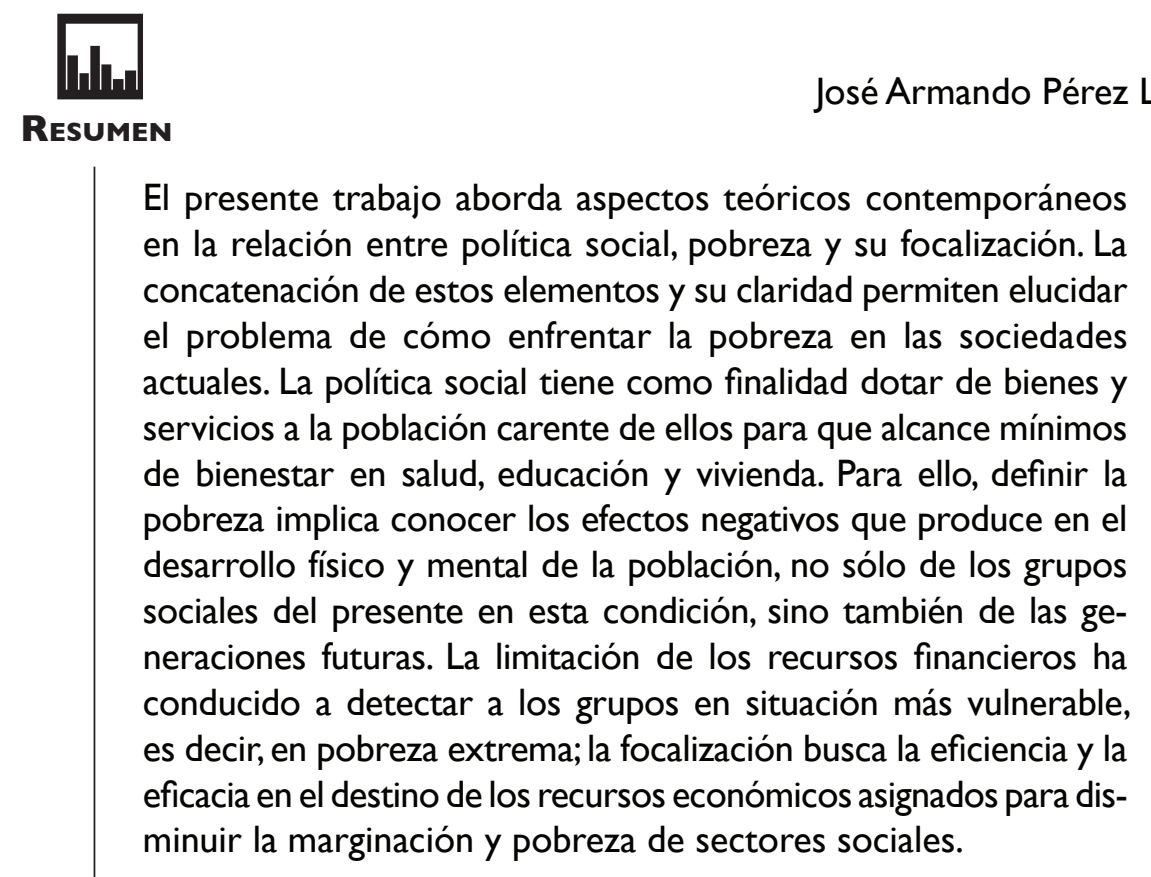

Palabras | Focalización, pobreza, pobreza absoluta, pobreza relativa, política CLAVE social.

*Universidad de Quintana Roo/jperez@uqroo.mx 
Política social,

pobreza y focalización

\section{Introducción}

El combate a la pobreza es uno de los retos primordiales de todos los gobiernos en el mundo, por lo que es necesario elaborar políticas sociales que sirvan a este propósito. La planeación del gasto permite ampliar la cobertura hacia los destinatarios y vigilar que los recursos lleguen rápida y eficientemente. De esta manera, se han diseñado diversos programas a nivel internacional respaldados por el Banco Mundial, que buscan llegar en forma directa a los grupos sociales que necesitan apoyos monetarios para elevar sus exiguos ingresos, en un esfuerzo por equilibrar los bajos niveles de consumo que los ubican en situación de vulnerabilidad nutritiva, educativa, laboral, de baja autoestima y de rechazo social. Bajo esta premisa, se abordan a continuación las características de la política social y de la pobreza destacando el papel que asume la focalización para combatir la pobreza en la que subsisten millones de personas.

\section{La política social}

A través de los diferentes marcos jurídicos se establecen las acciones de los gobiernos para la ejecución de medidas económicas y políticas que beneficien a la población en áreas de salud, vivienda, educación y alimentación, por mencionar algunos rubros. Esto lo identificamos como política social de una institución que gobierna a un conglomerado con necesidades y demandas crecientes en tiempo e ineluctables. Sin embargo, podemos anotar que:

un primer problema es aclarar y definir el concepto de política social; puesto que no existe uno solo que sea universalmente aceptado y que abarque todas las acciones que pueden caer dentro del amplio campo de lo social. El supuesto conceptual recupera la idea de expresar a la política social en un sentido amplio. Podría decirse que el móvil de la política social consistiría en el deseo de asegurar a todos los miembros de la sociedad unos niveles mínimos de bienestar y, en todo caso, ciertas oportunidades para acceder a esos mínimos [Pardo, 2000:46I].

Vista la política social como una generalidad, parecería que los objetivos, las acciones y metas establecidas en los planes y programas tienen como condición indispensable tratar en la misma magnitud e igualdad a toda la población. Sin embargo, en la política social existen apartados específicos que indican la vulnerabilidad de grupos sociales que por condiciones históricas, 
geográficas, étnicas y educativas, principalmente, no están en situación de recibir y disfrutar los beneficios señalados, por lo que se deben plantear estrategias que les permitan el acceso.

En este sentido, la utilización de las políticas sociales con fines partidistas es motivo de controversia. Para conservar el poder en las sociedades democráticas, la clase política gobernante tiende a coaccionar a la ciudadanía en edad de votar supeditando el suministro de servicios a una preferencia partidista. Este tipo de prácticas desvirtúa los objetivos, no beneficia a la población que necesita de las políticas sociales e impide que los recursos sean aprovechados en su totalidad. La primera tarea para corregir esta práctica consiste en diseñar y aplicar mecanismos que legitimen ante la sociedad la imparcialidad de las políticas públicas (Mendoza, 200I)

Los grupos sociales marginados de los beneficios del desarrollo económico son sujetos de políticas sociales que pretenden dotarlos de servicios que cubran sus necesidades de desarrollo físico e intelectual: educación, vivienda, salud y alimentación; para lograrlo, es necesario establecer la equidad y eficiencia como fines de las políticas sociales; Cohen y Franco (2003) distinguen las siguientes:

a) Las que buscan elevar el nivel de consumo de una parte de la población (que algunos llaman asistenciales y paliativas) se rigen en todo momento por el principio de equidad. Cabe destacar, empero, que es muy común que el consumo de los grupos pobres lleve en sí un fuerte componente de inversión en capital humano, puesto que de no generarse ese consumo se producirían daños en las personas de esos grupos y constituirían, inevitablemente, una descapitalización.

b) La inversión en recursos humanos, obviamente, tiene un horizonte de mediano y largo plazo y responde tanto a las necesidades de la clientela, que requiere capital humano para satisfacer por sí misma sus necesidades, como a demandas de la sociedad que exige una población en condiciones de asegurar su continuidad y desarrollo. El principio de equidad rige estas acciones en lo que respecta a corto plazo y a la clientela, mientras que el principio de eficiencia predomina en el mediano y largo plazo, y en relación con los intereses sociales.

Se puede señalar que la política social tiene como principio construir mecanismos de equidad que sean sujetos a la evaluación del mismo conglomerado 
Política social,

pobreza y focalización

y, desde luego, que combatan los accesos segmentados de grupos políticos, la universalidad aparente, la regresividad, el tradicionalismo y la inercia.

\section{Caracterización de la pobreza}

El tema de la pobreza ha sido un punto nodal sobre el que organismos internacionales, gobiernos, académicos e investigadores han hecho diferentes interpretaciones. La división mundial en países pobres y ricos, desarrollados y subdesarrollados, centrales y periféricos, sólo son formas de indicar las diferencias en la calidad de vida de la población distribuida en el orbe contemporáneo. Lo que sí queda claro es que independientemente del eufemismo que se utilice, la utilización y racionalización de los recursos naturales, el crecimiento de las actividades económicas con sustento en el avance científico y tecnológico, la preparación del capital humano y la participación de los actores sociales en las dinámicas propias de cada país ocurren con una gran desventaja para las naciones contextualizadas como pobres.

Ante este panorama, para definir la pobreza de las personas o de una sociedad, los estudiosos del tema han tenido que consensuar sus puntos de vista, aun cuando ello implique que la metodología usada quede sujeta a la duda y reservas del caso; a lo cual se suma la interpretación que hacen de la pobreza las concepciones teóricas y el bagaje conceptual afin a ésta que elaboran.

Los conceptos de estudio de la pobreza comienzan con la identificación de necesidades y preferencias o utilidad del individuo frente a la elección de los bienes a consumir. En las necesidades podemos identificar carencias de bienes fundamentales para la sobrevivencia humana, mientras que las preferencias por el consumo, de acuerdo con la utilidad que se busca, están influenciadas por la ideología contemporánea del libre mercado y el supuesto de la libre elección del consumidor. De este modo: "El desplazamiento de las necesidades por las preferencias permite justificar plenamente el dominio del mercado sobre la política" (Boltvinik, 2003:4I0). La confrontación de los conceptos es el primer obstáculo a salvar; en este trabajo aceptamos que las necesidades no son cubiertas por las leyes del mercado, donde la preferencia por la ganancia es lo primordial y fundamento de su esquema teórico-ideológico. Asimismo, Boltvinik señala que es necesario hacer una aclaración de lo que se entiende por necesidades e indica que éstas las podemos separar en: 
a) Necesidades por impulso o pulsión, que proviene de los deseos de los individuos y no necesariamente tienen que ver con la salud física, mental o de bienestar (por ejemplo, fumar).

b) Las necesidades humanas que corresponden a metas y propósitos universales cuyo objetivo de vida pública es preservarla y mejorarla (alimentación, ropa, vivienda, etcétera).

De esta manera, el objetivo primordial es la satisfacción de las necesidades humanas para el desarrollo de una sociedad con prospectiva. Boltvinik indica como ineluctable que existan criterios que contribuyan de manera universal a la salud física y a la autonomía de los individuos, por lo que propone las siguientes características que deben poseer los satisfactores, ya que:

[se] asocian las necesidades a la prevención del grave daño que se presentaría si no se satisfacen, esté o no consciente de ello el individuo [...] I) Comida nutritiva y agua limpia, 2) vivienda protectora, 3) medio de trabajo no dañino, 4) medioambiente no perjudicial, 5) adecuada atención a la salud, 6) seguridad en la niñez, 7) relaciones primarias significativas, 8) seguridad física, 9) seguridad económica, 10) educación apropiada, II) control natal y partos seguros [Boltvinik, 2003:4II].

La puntualización de lo deseable para el bienestar de la población y de los requerimientos que debe cubrir o garantizar el Estado son elementos de la justicia social (Rawls, 1979), en el entendido de que la fortaleza radica en tener clara la práctica del gobierno con los grupos subalternos, como sujetos de participación en el diseño de las políticas sociales.

Además, la justicia distributiva está formada por varios elementos. Para empezar, es interesante conocer la concepción que se tiene de los sujetos que reciben los bienes y los servicios; por ejemplo [...] si se considera que los pobres son seres pasivos, ignorantes de sus necesidades, e incapaces de elegir aquello que más les conviene, se implementarán políticas paternalistas. Por el contrario, si se le considera agentes activos, se apoyaran aquellas políticas que les permitan formar sus propios planes de vida [Dieterlen, 2003:14].

Por lo tanto, se propone la proactividad de los grupos pobres.

La existencia de grupos sociales cuyo nivel de consumo no les permite alcanzar el pleno desarrollo de sus capacidades físicas e intelectuales, y que 
Política social,

pobreza y focalización

van quedando a la zaga de los beneficios de progreso social, nos dan la pauta para retomar lo siguiente:

Podemos llegar a una definición de pobreza partiendo de la establecida por la Comisión de las Comunidades Europeas en el Reporte de las acciones específicas comunitarias sobre los programas para combatir la pobreza. La definición es la siguiente: "Pobres son aquellas personas, familias y grupos de personas cuyos recursos (materiales, culturales y sociales) son tan limitados que los obligan a estar excluidos de una forma de vida mínima aceptable en los Estados en que viven" [Dieterlen, 2003: 27].

Por su parte, el Banco Mundial (2004: I) indica:

la falta de ingresos es sólo una medida del bienestar de los grupos en desventaja. La pobreza tiene muchas dimensiones: condiciones educativas y de salud inadecuadas, falta de acceso a los servicios básicos, vulnerabilidad, baja autoestima y exclusión social [...] entre los factores estructurales se encuentran patrones de propiedad de activos, geografía e influencias institucionales formales e informales que proceden de fuentes políticas, sociales y culturales. Entre las influencias externas de corto plazo están los efectos del contexto económico internacional sobre economía, los patrones de precios y las oportunidades de empleo, así como las afectaciones relacionadas con el clima.

De esta manera,Vélez rescata la siguiente clasificación:

Volviendo a Rawls, con el objeto de saber quiénes son los individuos menos favorecidos es necesario distinguir entre la pobreza moderada y la extrema,ya que los individuos ubicados en esta última categoría son los que deben ser beneficiados por los principios de justicia. Así, los pobres extremos son las personas que no tienen un nivel de nutrición suficiente, lo cual incide en un desempeño físico y mental deficiente que no les permite participar en el mercado de trabajo ni en actividades intelectuales, como la educación. Además, la condición de pobreza extrema prácticamente impide cualquier movilidad social; los pobres extremos nacen y mueren por lo general en la misma situación. Por otra parte, los pobres moderados son los que, debido al grado de desarrollo de un país en un momento dado, no cubren lo que se considera como necesidades básicas. Se diferencian de los pobres extremos en que sí tienen la capacidad, pero no las oportunidades de participar en actividades económicas e intelectuales [...] Los habitantes en extrema pobreza requieren medidas inmediatas para salir de la marginación y para reducir su debilidad y su vulnerabilidad al medio [Vélez, 1994: 9]. 
Ahora bien, según Santiago Levy (citado por Dieterlen, 2003: 29) se identifican siete características en la situación de las personas que se encuentran en estado de pobreza extrema:

I) Tienen una tasa más alta de fecundidad.

2) No pueden responder a una baja transitoria de salarios. No les es posible trabajar más de lo que hacen y, por lo tanto, no pueden compensar los tiempos de depresión económica.

3) Los miembros de las familias que se encuentran en una situación de pobreza extrema tienen una tasa más alta de participación en la vida económica y los hijos participan desde temprana edad, por esta razón, el costo de la educación es demasiado alto.

4) Es menor su capacidad para enfrentar los riesgos, ya que tienen amenazas nutricionales inducidas por la caída del ingreso. Con poco acceso al crédito y pocos activos comercializables, las reducciones en el ingreso se traducen de inmediato en consumo menor.

5) La dieta cambia según la elasticidad del precio de los alimentos y del ingreso.

6) El nivel nutricional tiene un efecto directo en la productividad, tanto en el caso de los adultos como en el de los niños.

7) Finalmente, la importancia de la desigualdad dentro de las familias es mayor en los hogares que se encuentran en una situación de pobreza extrema, ya que ésta determina cómo los recursos adicionales para la familia como un todo se traducen en recursos para cada uno de sus miembros.

De esta manera, el uso de estadísticas oficiales para la aceptación de los grupos en pobreza extrema debe partir de procedimientos claros. La situación de la pobreza extrema se detecta por varios indicadores: la composición del hogar por edad y sexo; el analfabetismo; la actividad económica y el ingreso de las familias; la presencia de minusválidos; el acceso a los servicios básicos, a los bienes $y$ a la propiedad de tierra y animales. En cuanto a los ingresos, se considera que una familia se encuentra en situación de pobreza si su ingreso per cápita es insuficiente para adquirir la canasta alimentaria normativa; otra manera de medir el grado de pobreza extrema es a partir de los niveles de desnutrición 
Política social,

pobreza y focalización

Para comparar la pobreza entre países, se utiliza la clasificación entre pobreza relativa y absoluta. En esta interpretación se hace énfasis en las formas de consumo determinadas por condiciones histórico-culturales, detectadas por los organismos internacionales. Robert MacNamara, cuando fue presidente del Banco Mundial, destacó la importancia de distinguir entre la pobreza absoluta y la relativa, donde la segunda se refiere a la que existe en las naciones industrializadas, en las que algunos ciudadanos son pobres en comparación con sus vecinos. Las personas que viven en un estado de pobreza relativa en Australia pueden estar mejor que los pensionados en Gran Bretaña,y éstos no son pobres si los comparamos con los que existen en Malí o Etiopía. La pobreza absoluta, por otro lado, es pobreza según cualquier estándar. En palabras de MacNamara:

La pobreza en un nivel absoluto [...] es una vida en los niveles ínfimos de la existencia. Los pobres absolutos son seres humanos con graves carencias, que luchan por sobrevivir en un ambiente de circunstancias miserables y degradantes casi más allá de lo que puede concebir el más sofisticado poder de la imaginación. Comparados con aquellos afortunados que viven en los países desarrollados, los que viven en los países pobres tienen una tasa de mortalidad ocho veces mayor, una expectativa de vida un tercio más baja, una tasa de analfabetismo sesenta por ciento mayor. Uno de cada dos miembros de la población se encuentra por debajo de los niveles nutricionales aceptables, y millones de niños pequeños consumen menos proteínas de las que se necesitan para un desarrollo óptimo del cerebro [Dieterlen, 2003: 31-32].

Por consiguiente, la pobreza relativa se define en relación con otras situaciones y contextos sociales con los cuales es confrontada y diferenciada, como también lo son sus grados y heterogeneidad, $y$ el análisis remite a privaciones mayores o menores derivadas de la comparación. En tanto, la pobreza absoluta alude a estados de carencia en los que se pasan por alto necesidades que todos, por compartir la calidad de seres humanos, tienen el derecho de satisfacer, razón por la cual no pueden ser relativizadas (Mendoza:200I). De esta manera, según Ziccardi (citado por Lozada, 2002: 56-58), las desventajas deben involucrar tres dimensiones: privación, exclusión y desigualdad económica, social y espacial:

a) Privación. Asociada a las condiciones de empleo que colocan a los trabajadores y a sus familias en una situación de precariedad. Medida a través del ingreso global en relación con lo necesario para acceder a un conjunto de bienes básicos. 
b) Exclusión. Se refiere a las dificultades de acceder a los bienes básicos (alimentación, salud y educación), lo cual coloca al trabajador y a su familia en una situación de exclusión social directa, como un conjunto de mecanismos que son considerados factores de riesgo social que agravan la situación de pobreza o impiden su superación, tales como: discriminación étnica y de género, los obstáculos para acceder al crédito, a la justicia, vivienda y servicios básicos, a las instancias de decisión gubernamental y política.

c) Desigualdad económica, social y espacial. Existentes entre el campo y la ciudad, entre regiones ricas y regiones pobres, en las condiciones en las que viven algunos sectores de la población y la constante miseria de las mayorías; desigualdad en el empleo y en los bienes y servicios, producto de las condiciones desventajosas en las que se integran los indígenas, las mujeres, los jóvenes que no acceden a niveles adecuados de educación y capacitación.

\section{Programas sociales y el combate a la pobreza}

Compartimos que la noción de pobreza absoluta es indispensable para plantear los programas políticos, especialmente en los países que enfrentan una profunda insatisfacción de necesidades básicas, independientemente de las diferencias culturales o sociales. A continuación veremos que la pobreza absoluta se encuentra íntimamente relacionada con dos factores que la colocan en un círculo vicioso: la desnutrición y el aumento de la tasa de nacimientos.

En esta interpretación, se continuó apuntalando la propuesta de elaboración de programas sociales que fueran más allá de la transferencia monetaria hacia estos grupos clasificados como pobres: "es importante tener en cuenta que la relación entre desnutrición e ingreso no siempre es directa. Un aumento en el ingreso no necesariamente implica una disminución de la desnutrición. Por esta razón es necesario que la desnutrición sea atacada directamente mediante complementos alimenticios" [Dieterlen, 2003: 34].

En el argumento de que el fenómeno de la desnutrición puede causar dos efectos, el primero se refiere al valor que una buena alimentación tiene en sí misma; una buena nutrición es deseable porque proporciona salud física, mental y mayor resistencia a la enfermedad. La alimentación también está ligada a la 
Política social,

pobreza y focalización

utilidad que proporciona, principalmente en un sentido funcional; eleva la capacidad de trabajo y, por lo tanto, la posibilidad de obtener ingresos. El segundo efecto tiene que ver con las preferencias que manifiestan los individuos por ciertos alimentos, ya sea porque tienen un buen sabor, porque se comercializan de manera efectiva o, aparentemente, su consumo refleja un incremento de prestigio social y económico. Así, es necesario atacar el problema de la causa de la desnutrición desde estos efectos.

La pobreza se refiere, entonces, a la insuficiencia de ingresos (flujos en dinero o en bienes) y de riqueza en el sentido de disponibilidad de activos. La debilidad física se vincula con la falta de fuerza, desnutrición, salud deficiente, incapacidad física y con una alta tasa de dependencia al grupo de pertenencia. El aislamiento se considera la lejanía física, la carencia de educación, la ignorancia y la falta de acceso a servicios e información. Vulnerabilidad se relaciona con tensión interna y externa y el peligro de volverse más pobre y carente de todo, mientras que en ello subsiste la falta de poder, entendido como la incapacidad y debilidad para enfrentar las arbitrariedades y exigencias de los poderosos u empleadores.

La interpretación de la pobreza es en sí vasta y compleja. Reconocerla como una situación lacerante de las sociedades contemporáneas representa un avance actual, pues soslayarla implicaría un desplome de las condiciones de vida que a futuro provocarían el deterioro profundo del tejido social y sociedades cada vez más tendientes a la inestabilidad y descontento general. Esto probablemente generaría un clima de violencia y más atraso tanto en lo económico como en lo político. Es en este sentido que se impulsa y se propone, junto con gobiernos locales, que se atienda la pobreza dentro de los márgenes que permita la política económica neoliberal, hoy en boga, sin menoscabo del mercado.

De esta manera, a partir de programas como Oportunidades, en México; Erradicación de Trabajo Infantil, en Brasil; Red de Protección Social, en Nicaragua, y Familias en Acción, de Colombia (Villatoro, 2004), por mencionar algunos deAmérica Latina con el reconocimiento y apoyo del Banco Mundial, se ha generado que los países enfrentan a la pobreza en los siguientes aspectos:

a) Un programa de apoyo a las familias pobres, que les facilite alcanzar niveles adecuados de alimentación, salud y educación, y permita así romper el círculo vicioso de la pobreza extrema.

b) Una política de empleo encaminada a mejorar las oportunidades de ingreso de las familias en pobreza extrema, que a su vez debe considerar: 
i) un conjunto de reformas en materia laboral y de seguridad social, que elimine obstáculos al crecimiento del empleo en el sector formal de la economía y permita un acceso fluido para todos los trabajadores, aun los poco calificados, y ii) un programa de empleos temporales que permita ocupar a los trabajadores más pobres de las zonas rurales, en la construcción de activos permanentes, en beneficio de la microregión.

c) Un decidido impulso a la inversión de infraestructura social en los municipios con mayores problemas de pobreza.

\section{Propuesta para disminuir el número de pobres en el mundo}

\section{La focalización de la pobreza}

Identificar a los grupos humanos en condiciones socioeconómicas desfavorables con respecto al resto de la población permite aprovechar al máximo los recursos destinados para combatir la pobreza. El reto de los gobiernos es modificar las condiciones de estos sectores sociales, vulnerables por condiciones geográficas, climatológicas, nutritivas, educativas, de vivienda, de ciclos económicos adversos frente a los que quedan inermes, de falta de capacitación para incorporarse a un mercado laboral cambiante. En este sentido, la focalización de estos grupos permite elaborar políticas especificas para hacerlos destinatarios directos. Asimismo, se sugiere disminuir la burocratización, evitar la duplicidad, orientar hábitos de consumo propicios para el desarrollo físico y mental saludables, así como involucrar a estos grupos en la toma de decisiones.

Se plantean dos argumentos para el desarrollo de la política focal de la pobreza: el de las crisis económicas y el de la focalización permanente. En el primer argumento, se señala la limitación de los recursos, pues durante una crisis económica se agudiza la escasez como consecuencia de la contracción del gasto público en general y del gasto social en particular. Esto conduce al incremento de las necesidades insatisfechas en la población y en especial de los grupos en pobreza extrema. La focalización permitiría la ayuda inmediata y la canalización de recursos para contribuir a la estabilidad social en estas regiones. En el segundo argumento, se justifica la focalización permanente, pues permite mejorar el 
Política social,

pobreza y focalización

diseño de programas. Cuánto más precisa sea la identificación de las carencias por satisfacer y sus destinatarios será más fácil diseñar medidas diferenciadas y específicas.Asimismo, aumentará la eficacia del programa y éste no se reduciría a épocas de crisis, pues en caso de ocurrir, sólo se tendrían que hacer ajustes con base en criterios que permitan alcanzar los objetivos prioritarios en la reducción de la pobreza (Rojas, 200I).

Ambos criterios aluden a la cuestión temporal y a la prevención de los ciclos económicos. Sin embargo, con la focalización de la pobreza se pretende identificar a los pobres en el ámbito regional, por nivel de ingresos, por carencias alimenticias y necesidades de salud. Pragmáticamente, se indican como objetivos principales de la focalización los siguientes puntos (Contreras, 2000: 498):

I) Atender con precisión la satisfacción de necesidades de personas excluidas cuantitativa y cualitativamente de satisfactores mínimos o esenciales, por razones culturales, socioeconómicas y de costos de transacción (transporte, tiempo). Este objetivo se refiere a las carencias por satisfacer.

2) Identificar con precisión a los beneficiarios potenciales. Este objetivo se refiere a la población-objetivo más vulnerable.

3) Diseñar programas para asegurar un alto impacto per cápita en el grupo seleccionado, mediante transferencias monetarias o la entrega de bienes o servicios.

La identificación de los actores participantes y su relación con los medios organizacionales permite establecer vínculos a través de normas y reglas de operación de los programas. De esta manera, el grupo-objetivo establece una relación con la lógica interna del programa, sus objetivos y, de ello, el aprendizaje de las reglas del juego (Cardozo, 2003a). La gestión pública valida así el sistema social e ideológico que le da sustento a las políticas públicas.

\section{Conclusión}

La pobreza extrema en la que viven grupos sociales marginados del crecimiento económico en diversos países del orbe, los enfrenta a un entorno de competencia en la que no tienen posibilidades de luchar en igualdad de condiciones. La 
desnutrición, el analfabetismo, la carencia de servicios médicos -incluyendo el acceso a medicamentos-, la falta de ingresos que les garantice un nivel básico de consumo para cubrir sus necesidades, y viviendas vulnerables, son el común denominador que permea su vida cotidiana. Estas necesidades no cubiertas se reflejan en baja autoestima, indefensión ante arbitrariedades laborales y de funcionarios públicos; es decir, la exclusión social por factores históricos, geográficos, culturales y económicos.

Por lo tanto, la disminución de la pobreza en el mundo es un reto para los gobiernos. Los mecanismos para disminuir el rezago social de estos grupos sociales deben provenir de organismos gubernamentales que impulsen y diseñen políticas sociales acordes con su entorno socioeconómico. Liberar estas políticas del clientelismo partidista y de intervencionismo de funcionarios públicos es fundamental. Sólo así se puede garantizar que los destinatarios sean copartícipes de la elaboración de sus demandas.

La focalización, instrumento para detectar a los grupos sociales carentes de los mínimos de bienestar, permite diseñar procesos para hacer llegar los apoyos en educación, salud, vivienda y alimentación que demandan los grupos marginados.

La elaboración de políticas sociales con una clara conceptualización de la pobreza y sólida focalización permitirá disminuir las condiciones de pobreza extrema de los grupos destinatarios. Esto llevará años, comenzar es apremiante. El conflicto social es una posibilidad latente.

\section{FUENTES CONSULTADAS}

Banco Mundial (2004).“La pobreza en México:una evaluación de las condiciones, las tendencias y la estrategia de gobierno” [en línea]. México, 28 de julio de 2004. Disponible en: http://www.bancomundial.org.mx

Boltvinik, Julio (2003).“La teoría de las necesidades humanas de Doyal y Gough”, Comercio Exterior. México, núm. 5, 4I 0-4I 2.

Cardozo Brum, Miriam (2003a).“El marco teórico-conceptual de la evaluación 
Política social,

pobreza y focalización

de las políticas públicas" [en línea]. Disponible en: http://www.cddhcu. gob.mx/biblot [2003]

22 (2003b).“Evaluación de políticas de desarrollo social”. Política y Cultura, núm. 20, I39-I54.

Cohen, Ernesto y Rolando Franco (2003). Evaluación de proyectos sociales. México: Siglo XXI

Contreras Suárez, Enrique (2000).“Una visión crítica de los programas actuales de combate a la pobreza", en Rolando Cordera y Alicia Ziccardi (coords). Las políticas sociales de México al fin del milenio. Descentralización, diseño gestión. México: UNAM/ ILS / FE / Porrúa, 497-5I0.

Dieterlen, Paulette (2003). La pobreza: un estudio filosófico. México: FCE

Lozada Vázquez (2002). El papel de Progresa en la reproducción de unidades domésticas campesinas. México: UNAM/ $\mathrm{CIICH} /$ Sedesol.

Mendoza Álvarez, Jorge José (200I). La reforma del Estado, política social, la condición de pobreza y desarrollo en México (1982-2000). Tesis de licenciatura. México: UNAM / Facultad de Economía.

Pardo, María del Carmen (2000). “El diseño administrativo de los programas de emergencia”, en Rolando Cordera y Alicia Ziccardi (coords.). Las políticas sociales de México al fin del milenio. Descentralización, diseño y gestión. México: UnAM/IIs/FE/ Porrúa, 459-496.

Rawls (1979). Teoría de la justicia. México: FCE.

Rojas Arciniega, Miguel Ángel (200I). Los programas de combate a la pobreza en México. Tesis de licenciatura. México: unam/ Facultad de Economía.

Vélez, Félix (1994). La pobreza en México. Causas y políticas para combatirlas. México: FCE.

Villatoro, Pablo (2004). Programas de reducción de la pobreza en América Latina. Un análisis de cinco experiencias. Santiago de Chile: CEPAL (Serie Políticas Sociales). 